ISSN 1112-9867

Available online at

http://www.jfas.info

\title{
IMPACT OF ZOOTECHNICAL PARAMETERS ON CELL QUALITY OF CATTLE MILK (SEMI-ARID COSTAL TUNISIA)
}

\author{
R. Haj Mbarek* and Y. M'Sadak
}

Institut Supérieur Agronomique de Chott Mariem- CP 4042, Université de Sousse, Tunisie

Received: 16 April 2015 / Accepted: 01 August 2015 / Published online: 1 September 2015

\begin{abstract}
The work was realized on a sample of 50 cattle herds, conducted in aboveground, in a coastal area of the semi-arid Tunisia, by using investigation related to cows and their breeding and milking conditions as well as despoliation in milk control data. Analysis of the data relating to the parameters of udder conformation and cow cleanliness revealed that "Udder depth" settings "Udder cleanliness" have been shown to affect the ICC and are considered factors risk of bovine mastitis. The study of breeding and milking conditions highlight some significant factors on changes on the variation of cell counts and the probability of the spread of mastitis, especially the "No disinfection of teat." The level of housing, the analysis revealed that the use of a litter reduced to half the average of ICC which proved highly related to the cleanliness of both the sleeping area as the udder.
\end{abstract}

Keywords: Cell counts; Udder conformation; Clean cows; Breeding and milking conditions; Semi-arid Tunisia.

Author Correspondence, e-mail: haj_mbarek_rim@yahoo.fr

doi: http://dx.doi.org/10.4314/jfas.v7i3.4

\section{INTRODUCTION}

L'élevage bovin laitier est régulièrement confronté à des mammites. 20\% des pathologies rencontrées chez la vache laitière constituent des infections mammaires [1]. Plus récemment, on a estimé que $30 \%$ des vaches laitières sont réformées à cause des mammites [2]. La mammite est une maladie de production qui présente diverses conséquences non seulement pour le producteur, mais également pour le consommateur de lait et de ses dérivés [3]. C'est la 
pathologie la plus coûteuse, car elle est la principale source de dépenses en soins vétérinaires et en médicaments $[4,5,6,7,8,9,10]$. L'infection mammaire est accompagnée par des élévations des comptages cellulaires individuels (CCI) des vaches laitières. Ainsi, une augmentation des CCI pourrait être associée à un risque d'apparition des mammites [11]. Ces élévations cellulaires génèrent des pertes quantitatives en lait [12, 13] jusqu'à 40\% [13], en grande partie, conséquentes aux mammites subcliniques [14, 13], détectables généralement par le dénombrement cellulaire somatique du lait $[15,16,17,18,19,3]$. En effet, les mammites de traite sont des mammites dont les germes responsables ne manifestent pas le plus souvent des signes cliniques, dans $60 \%$ des cas l'infection reste subclinique [20]. Plusieurs études avaient comme but la mise en évidence des facteurs de risque liés à cette maladie [21, 22, 23, 24]. Les résultats diffèrent souvent en fonction de la variation des conditions d'étude.

Le présent travail se propose une analyse descriptive et analytique de l'effet de certains paramètres zootechniques (conformation des mamelles, propreté des vaches, conditions d'élevage, équipements et pratiques de traite) sur les CCI du lait en élevage bovin hors sol dans la région de Sousse, zone côtière semi-aride de la Tunisie.

\section{MATÉRIEL ET MÉTHODES}

L'étude entreprise a été réalisée à partir des données recueillies par le contrôle laitier relatif à 50 élevages totalisant 661 vaches présentes dont 535 vaches en lactation (parmi les 127 élevages contrôlés) dans le gouvernorat de Sousse en Tunisie. 297 vaches de race Pie Noire Holsteinisée en lactation ont été retenues pour cette investigation.

La répartition des exploitations dans la région d'étude est au hasard. On a regroupé les exploitations en trois groupes suivant leur localisation régionale. La distribution des élevages et leurs effectifs en vaches laitières sont relatés dans le tableau 1.

Il convient de signaler que la délégation Kalâa Kébira, berceau laitier de la région de Sousse, a été bien représentée avec $48 \%$ des élevages considérés réunissant $40 \%$ des vaches présentes (VP), $40 \%$ des vaches en lactation (VL) et $46 \%$ des vaches considérées (VC).Tous les troupeaux étudiés sont soumis à la traite mécanique.

Ces troupeaux sont conduits selon le système hors sol, caractérisé par le manque, voire l'absence de surface fourragère disponible, en raison d'une pluviométrie annuelle insuffisante ne dépassant pas les $350 \mathrm{~mm}$ (milieu semi-aride) et des ressources en eau généralement de mauvaise qualité (salinité élevée). L'étude a été conduite à partir du dépouillement des données cellulaires réunies auprès de l'Office d'Elevage et des Pâturages (OEP) de Sousse. 
Tableau 1. Répartition géographique des exploitations suivies et effectifs des vaches

\begin{tabular}{|c|c|c|c|c|c|c|c|c|c|}
\hline \multirow{2}{*}{$\begin{array}{c}\text { Zone } \\
\text { Sous-zone }\end{array}$} & \multicolumn{3}{|c|}{$\begin{array}{c}\text { Zone 1 : } \\
\text { Sousse Nord } \\
\end{array}$} & \multicolumn{3}{|c|}{$\begin{array}{c}\text { Zone 2 : } \\
\text { Sousse Centre }\end{array}$} & \multicolumn{2}{|c|}{$\begin{array}{c}\text { Zone 3 : } \\
\text { Sousse Sud }\end{array}$} & \multirow[t]{2}{*}{ Total } \\
\hline & Enfidha & Hergla & $\begin{array}{c}\text { Sidi } \\
\text { Bou } \\
\text { Ali }\end{array}$ & $\begin{array}{l}\text { Kalaa } \\
\text { Kébira }\end{array}$ & Akouda & $\begin{array}{c}\text { Hammam } \\
\text { Sousse }\end{array}$ & $\begin{array}{c}\text { Kalaa } \\
\text { Sghira }\end{array}$ & Msaken & \\
\hline $\begin{array}{l}\text { Nombre } \\
\text { d'élevages }\end{array}$ & 2 & 3 & 6 & 24 & 1 & 2 & 2 & 10 & 50 \\
\hline $\begin{array}{l}\text { Vaches } \\
\text { présentes }\end{array}$ & 24 & 64 & 128 & 260 & 26 & 34 & 25 & 100 & 661 \\
\hline $\begin{array}{l}\text { Vaches en } \\
\text { lactation }\end{array}$ & 22 & 55 & 99 & 216 & 14 & 28 & 23 & 78 & 535 \\
\hline $\begin{array}{c}\text { Vaches } \\
\text { considérées }\end{array}$ & 10 & 27 & 44 & 136 & 4 & 15 & 15 & 54 & 297 \\
\hline
\end{tabular}

Les données extraites datent du début du mois de Janvier 2009 jusqu'à la fin du mois d'Avril 2011 (28 mois de contrôle) pour garantir un nombre maximal de vaches contrôlées et ayant au moins une lactation complète. Les vêlages considérés sont ceux appartenant à l'intervalle $\left[1^{\mathrm{er}}\right.$ Janvier 2009-30 Avril 2010]. On s'est intéressé à dix contrôles successifs de la lactation considérée pour chaque vache.

Pour l'analyse des données, on a utilisé comme variables individuelles, celles relatives à la conformation des mamelles (profondeur du pis, qualité des attaches, position des trayons, taille et forme des trayons) et à la propreté : du pis, des flancs et des cuisses et des pattes arrière. Pour chaque variable, on a fixé quatre modalités et on a calculé la moyenne des CCI relative à chaque modalité. Lors du suivi, on a utilisé une fiche de notation de la conformation mammaire et de la propreté des vaches et une fiche de contrôle des conditions d'élevage et de traite. La notation de la conformation et de la propreté avec le contrôle de l'existence éventuelle des anomalies des trayons ont été accomplis en se basant sur les principes utilisés par Simon \& Jean Philippe [25].

Après la collecte des valeurs CCI à partir des fiches de contrôle laitier, on a accompli une enquête sur les conditions d'élevage (stabulation, litière, ...) et de traite (équipement et chantier) pour chaque troupeau, en faisant appel à la fiche élaborée à cet effet.

Concernant le traitement des données relatives à l'étude des facteurs de risque liés aux mammites, on a adopté, pour la mise en évidence des corrélations éventuelles, la procédure GLM du logiciel Statistical Analysis Système (logiciel SAS, version 9.13) à l'aide du modèle linéaire généralisé pour l'analyse de la variance des différents contrôles considérés. L'édition des données a été effectuée à partir des tableaux des fréquences (procédures «FREQ» et MEANS »). 


\section{RÉSULTATS ET DISCUSSION}

\subsection{Considérations générales}

Lors de cette étude relative à dix contrôles laitiers successifs par vache, nous avons utilisé comme variables les paramètres concernant la conformation des mamelles et la propreté des vaches, certaines conditions d'élevage, ainsi que quelques conditions (équipements et pratiques) de traite, susceptibles d'être des facteurs de risque des mammites subcliniques.

Chaque variable considérée peut avoir deux modalités ou plus. A titre indicatif, pour la conformation de la profondeur du pis : Mauvaise/ Moyenne/ Bonne/ Très Bonne ; pour le type de stabulation : Entravée/ Libre ; pour la litière : existe/ n'existe pas ou bien pour le nettoyage de la mamelle : avec eau seulement/ avec une lavette/ avec du savon, ...

\subsection{Impact de la conformation mammaire et de la propreté des vaches sur les CCI}

\subsubsection{Effet de la conformation des mamelles}

Les caractères de conformation chez une vache et leur répartition en fonction des CCI sont illustrés dans le tableau 2.

On remarque que les deux extrêmes de qualification de la conformation (Mauvaise, Très Bonne) ont présenté les CCI moyens les plus élevés. Dans une étude visant la recherche de l'impact de la morphologie de la mamelle et des trayons sur la santé mammaire des vaches, Slettbakk et al. [26] ont rapporté qu'une diminution de la distance entre l'extrémité du trayon et le sol est significativement associée aussi bien à une élévation des CCI qu'à la survenue de mammites cliniques. Les résultats antérieurs obtenus par Bakken [27] vont également dans ce sens. Cette constatation a été expliquée par le fait qu'une mamelle basse (Pis Profond) est davantage exposée aux souillures et aux blessures qu'une mamelle bien accrochée [27]. Concernant les vaches ayant une très bonne conformation, généralement, ce sont des primipares. L'origine du niveau élevé des CCI observés chez les primipares serait liée aux cellules somatiques concentrées dans un faible volume de lait, à cause de la faible production laitière [28]. Les deux qualifications (Moyenne, Bonne) ont révélé toujours les moyennes les plus faibles pour les quatre caractères mammaires examinés.

Dans les conditions d'étude, l'analyse de la variance des CCI des contrôles considérés a montré que seul le paramètre «Profondeur Pis » est susceptible d'affecter les CCI, son effet est significatif pour la moitié des contrôles $(\mathrm{P}<0,05)$. 
Tableau 2. Relation entre CCI moyens (x1000 cell. /mL) et conformation mammaire

\begin{tabular}{|c|c|c|c|}
\hline Paramètre de conformation & Effectif & $\%$ & CCI moyens \\
\hline \multicolumn{4}{|l|}{ Profondeur Pis } \\
\hline Mauvaise & 36 & 12 & $1326 \pm 1722$ \\
\hline Moyenne & 57 & 19 & $880 \pm 1067$ \\
\hline Bonne & 201 & 68 & $856 \pm 1215$ \\
\hline Très Bonne & 3 & 1 & $1869 \pm 3429$ \\
\hline Total & 297 & 100 & \\
\hline \multicolumn{4}{|l|}{ Oualité Attaches } \\
\hline Mauvaise & 29 & 10 & $1435 \pm 1762$ \\
\hline Moyenne & 56 & 19 & $927 \pm 1175$ \\
\hline Bonne & 209 & 70 & $844 \pm 1197$ \\
\hline Très Bonne & 3 & 1 & $1869 \pm 3429$ \\
\hline Total & 297 & 100 & \\
\hline \multicolumn{4}{|l|}{ Position Travons } \\
\hline Mauvaise & 90 & 30 & $1106 \pm 1432$ \\
\hline Moyenne & 89 & 30 & $744 \pm 1137$ \\
\hline Bonne & 114 & 38 & $913 \pm 1200$ \\
\hline Très Bonne & 4 & 2 & $1423 \pm 2596$ \\
\hline Total & 297 & 100 & \\
\hline \multicolumn{4}{|l|}{ Taille et Forme Trayons } \\
\hline Mauvaise & 54 & 18 & $1160 \pm 1456$ \\
\hline Moyenne & 62 & 21 & $856 \pm 1141$ \\
\hline Bonne & 177 & 60 & $871 \pm 1229$ \\
\hline Très Bonne & 4 & 1 & $1423 \pm 2596$ \\
\hline Total & 297 & 100 & \\
\hline
\end{tabular}

La taille et la forme des trayons sont en relation avec la vitesse de traite, et par la suite, avec la facilité d'extraction du lait de la mamelle [29]. Les paramètres «Qualité Attaches » et «Position Trayons » sont non significatifs dans le contexte de cette étude. Le paramètre « Taille et Forme Trayons » est significatif seulement au premier et au septième contrôle.

La variation des résultats obtenus en fonction des contrôles pourrait être due aux interactions entre les différents facteurs de risque associés aux mammites au sein des troupeaux suivis. La situation d'un élevage oscille d'un contrôle à un autre, en raison de la multitude des facteurs mis en jeu pouvant affecter la situation sanitaire mammaire des vaches.

\subsubsection{Effet de la propreté des vaches}

La propreté des vaches est un élément d'appréciation de l'hygiène générale et constitue une synthèse concrète des souillures (apportées par le milieu) et des facteurs pathogènes qui leur sont liés [30]. Le tableau 3 montre que la «Bonne » propreté des mamelles, des pattes arrière et des flancs et cuisses est associée aux CCI moyens les plus faibles. Par contre, la qualification « Très Bonne » présente les CCI moyens les plus élevés. 
Tableau 3. Relation entre CCI moyens (x1000 cell. $/ \mathrm{mL})$ et propreté des vaches

\begin{tabular}{cccc}
\hline Propreté & Effectif & \% & CCI moyen \\
\cline { 1 - 3 } Mamelle & \multicolumn{3}{c}{} \\
Mauvaise & 86 & 29 & $828 \pm 1128$ \\
Moyenne & 66 & 22 & $1267 \pm 1700$ \\
Bonne & 143 & 48 & $803 \pm 1114$ \\
Très Bonne & 2 & 1 & $2211 \pm 3959$ \\
\cline { 1 - 3 } Total & 297 & 100 & \\
\cline { 1 - 3 } Pattes arrière & \multicolumn{3}{c}{} \\
Mauvaise & 155 & 52 & $866 \pm 1190$ \\
Moyenne & 56 & 19 & $1237 \pm 1855$ \\
Bonne & 84 & 28 & $805 \pm 965$ \\
Très Bonne & 2 & 1 & $2211 \pm 3959$ \\
\cline { 1 - 2 } Total & 297 & 100 & \\
\cline { 1 - 3 } Flancs et cuisses & \multicolumn{3}{c}{} \\
Mauvaise & 181 & 61 & $901 \pm 1230$ \\
Moyenne & 73 & 24 & $1098 \pm 1535$ \\
Bonne & 41 & 14 & $681 \pm 845$ \\
Très Bonne & 2 & 1 & $2211 \pm 3959$ \\
\cline { 1 - 2 } Total & 297 & 100 &
\end{tabular}

Les résultats de l'analyse de la variance des CCI des contrôles considérés ont révélé que la propreté de la mamelle est significative pour la moitié des contrôles $(\mathrm{P}<0,05)$.

La précédente constatation sur l'importante variation des résultats relatifs à la conformation mammaire reste également valable dans le cas de la propreté des vaches.

\subsection{Analyse descriptive des paramètres d'élevage et de traite des vaches}

\subsubsection{Variation des paramètres selon la zone d'étude}

On a constaté que les éleveurs d'une zone font appel à des pratiques d'élevage et de traite non adoptées chez les éleveurs des autres zones. Le tableau 4 illustre la répartition de ces pratiques en fonction de la zone. La stabulation libre est rencontrée davantage dans la zone 2 (42\%) avec l'absence de litière chez $46 \%$ des élevages de cette zone. $52 \%$ des élevages ne pratiquant pas l'essuyage des trayons se localisent dans la zone 2 avec $38 \%$ d'entre eux n'éliminent pas les premiers jets de lait. Dans la même zone, l'égouttage mécanique est adopté chez presque la moitié des élevages. Par ailleurs, la dépose des gobelets-trayeurs est effectuée par arrachage (pratique déconseillée) chez 58\% des élevages (38\% localisés dans la zone 2). Finalement, la désinfection des trayons après la traite est non admise par $82 \%$ des élevages dont $50 \%$ appartiennent à la zone 2 . 
Tableau 4. Variation des principales conditions d'élevage et de traite selon la zone d'étude

\begin{tabular}{|c|c|c|c|c|c|}
\hline \multicolumn{2}{|r|}{ Variables } & \multicolumn{3}{|c|}{$\%$ Elevages } & \multirow{2}{*}{$\begin{array}{c}\% \\
\text { Total }\end{array}$} \\
\hline & & $\begin{array}{c}\text { Zone } \\
1\end{array}$ & $\begin{array}{c}\text { Zone } \\
2\end{array}$ & $\begin{array}{c}\text { Zone } \\
3\end{array}$ & \\
\hline \multirow{3}{*}{$\begin{array}{l}\text { Conditions } \\
\text { d'élevage }\end{array}$} & Stabulation : Libre & 18 & 42 & 20 & 80 \\
\hline & $\begin{array}{c}\text { Propreté Aire de couchage : } \\
\text { Mauvaise }\end{array}$ & 2 & 6 & 6 & 14 \\
\hline & Existence Litière : Non & 16 & 46 & 24 & 86 \\
\hline \multirow{10}{*}{$\begin{array}{l}\text { Conditions de } \\
\text { traite }\end{array}$} & $\begin{array}{ll}\text { Etat } & \text { Manchons-Trayeurs : } \\
\text { Mauvais } & \\
\end{array}$ & 2 & 20 & 8 & 30 \\
\hline & État Tuyauterie : Mauvais & 0 & 6 & 2 & 8 \\
\hline & Lavage Mamelle: Eau seulement & 12 & 28 & 10 & 50 \\
\hline & Essuyage Mamelle : Non & 18 & 52 & 22 & 92 \\
\hline & Éliminations Premiers jets : Non & 14 & 38 & 14 & 66 \\
\hline & $\begin{array}{l}\text { Pose des Gobelets-Trayeurs : } \\
\text { Indirecte }\end{array}$ & 4 & 4 & 8 & 16 \\
\hline & Égouttage : Mécanique & 20 & 46 & 24 & 90 \\
\hline & Surtraite : Oui & 6 & 26 & 14 & 46 \\
\hline & $\begin{array}{c}\text { Dépose Gobelets-Trayeurs : } \\
\text { Arrachage }\end{array}$ & 6 & 38 & 14 & 58 \\
\hline & Désinfection Trayons : Non & 14 & 50 & 18 & 82 \\
\hline
\end{tabular}

\subsubsection{Variation des paramètres selon la taille des troupeaux}

D'après le tableau 5 , on constate que les élevages ayant un effectif $>10 \mathrm{VP}$, ont presque toujours les pourcentages les plus élevés des pratiques non recommandées présentées. En effet, 38\% des exploitations n'utilisent pas de litière pour couvrir l'aire de couchage et n'essuient pas les mamelles des vaches après le nettoyage avec seulement de l'eau dans $18 \%$ de ces élevages. Les élevages avec des effectifs allant de 6 à 10 VP montrent aussi quelques pratiques déconseillées comme l'égouttage mécanique prolongé (34\%), le non essuyage de la mamelle (34\%) et la non désinfection des trayons (34\%). Concernant les troupeaux ayant une taille $\leq 5 \mathrm{VP}$, certaines anomalies ont été observées, mais le degré est moindre par rapport aux autres troupeaux. A ce propos, on peut dire que les petits éleveurs s'occupent mieux de leurs troupeaux en s'orientant mieux vers les bonnes pratiques [31].

\section{4. Étude analytique des paramètres d'élevage et de traite des vaches}

\subsubsection{Impact des conditions d'élevage sur les CCI}

Les résultats de l'analyse de la variance des CCI des contrôles considérés en fonction des conditions d'élevage ont montré que la conduite en stabulation libre ne diffère pas beaucoup de celle en stabulation entravée dans les conditions d'étude. L'existence de la litière réduit à moitié les CCI moyens qui se sont avérés fortement liés à la propreté de l'aire de couchage (Tableau 6). Seule la propreté de l'aire de couchage est peu significative vers les derniers 
contrôles qui ont coïncidé avec la deuxième phase de la lactation. Une litière insuffisamment entretenue augmenterait les risques des mammites subcliniques [32].

Tableau 5. Variation des principales conditions d'élevage et de traite selon la taille des troupeaux

\begin{tabular}{|c|c|c|c|c|c|}
\hline \multicolumn{2}{|r|}{ Variables } & \multicolumn{3}{|c|}{ \% Elevages } & \multirow{2}{*}{$\begin{array}{c}\% \\
\text { Total }\end{array}$} \\
\hline & & $\begin{array}{l}\leq 5 \\
\mathrm{VP}\end{array}$ & $\begin{array}{l}\text { 6-10 } \\
\text { VP }\end{array}$ & $\begin{array}{l}>10 \\
\text { VP }\end{array}$ & \\
\hline \multirow{3}{*}{$\begin{array}{l}\text { Conditions } \\
\text { d'élevage }\end{array}$} & Stabulation : Libre & 16 & 30 & 34 & 80 \\
\hline & $\begin{array}{c}\text { Propreté Aire de couchage : } \\
\text { Mauvaise }\end{array}$ & 6 & 4 & 4 & 14 \\
\hline & Existence Litière : Non & 18 & 30 & 38 & 86 \\
\hline \multirow{10}{*}{$\begin{array}{l}\text { Conditions de } \\
\text { traite }\end{array}$} & $\begin{array}{l}\text { État Manchons-Trayeurs: } \\
\text { Mauvais }\end{array}$ & 6 & 16 & 8 & 30 \\
\hline & État Tuyauterie : Mauvais & 0 & 4 & 4 & 8 \\
\hline & Lavage Mamelle: Eau seulement & 10 & 22 & 18 & 50 \\
\hline & Essuyage Mamelle : Non & 20 & 34 & 38 & 92 \\
\hline & Éliminations Premiers jets : Non & 12 & 24 & 30 & 66 \\
\hline & $\begin{array}{l}\text { Pose des Gobelets-Trayeurs : } \\
\text { Indirecte }\end{array}$ & 4 & 6 & 6 & 16 \\
\hline & Égouttage : Mécanique & 20 & 34 & 36 & 90 \\
\hline & Surtraite : Oui & 10 & 18 & 18 & 46 \\
\hline & $\begin{array}{c}\text { Dépose Gobelets-Trayeurs : } \\
\text { Arrachage }\end{array}$ & 20 & 16 & 22 & 58 \\
\hline & Désinfection Trayons : Non & 18 & 34 & 30 & 82 \\
\hline
\end{tabular}

Tableau 6. Relation entre CCI moyens (x1000 cell. $/ \mathrm{mL}$ ) et conditions d'élevage

\begin{tabular}{cccc}
\hline Paramètre & Effectif & $\%$ & CCI moyens \\
\hline Stabulation & & & \\
Entravée & 44 & 15 & $901 \pm 1417$ \\
Libre & 253 & 85 & $930 \pm 1245$ \\
\hline Litière & & & \\
Existe & 48 & 16 & $547 \pm 606$ \\
N'existe pas & 249 & 84 & $1001 \pm 1398$ \\
\hline Propreté Aire de couchage & & & \\
Mauvaise & 34 & 12 & $1358 \pm 1837$ \\
Moyenne & 122 & 41 & $930 \pm 1339$ \\
Bonne & 96 & 32 & $866 \pm 1184$ \\
Très Bonne & 45 & 15 & $729 \pm 842$ \\
\hline
\end{tabular}

\subsubsection{Impact des conditions de traite sur les CCI}

\section{a. Équipements de traite et leur effet sur les CCI}

Certains auteurs ont rapporté que le non contrôle annuel de la machine à traire est associé à une augmentation de la fréquence des mammites subcliniques [33, 34, 35]. Le testage permet 
de corriger les paramètres de fonctionnement de la machine à traire, afin qu'ils respectent les normes et traumatisent le moins possible les trayons. Cela se traduirait par une baisse de la fréquence des mammites subcliniques et une meilleure numération cellulaire [36]. Un niveau de vide trop important, des pulsateurs déréglés (fréquence ou rapport de pulsation) et des manchons trop durs augmentent la sensibilité de la mamelle [37]. La surtraite et/ou l'arrachage des griffes à lait sans coupure du vide en fin de traite, diminuent les défenses de la mamelle [38, 39].

Pour les paramètres «Niveau de vide», «Fréquence de pulsation» et «Rapport de pulsation », on remarque que la qualification «Conforme» a toujours les CCI moyens les plus élevés, alors que pour le «Décalage » moindre $(<5 \%)$, on a enregistré les CCI moyens les plus faibles. La non signification des effets des conditions de traite considérées $(\mathrm{P}>0,05)$ a été relevée pour l'ensemble de quatre paramètres examinés (Tableau 7). Cette situation relevée ne coïncide pas avec ce qui est annoncé dans la littérature [40, 41]. Un tel résultat a été obtenu suite à la multitude des facteurs mis en jeu et des corrélations multiples, difficiles à mettre en œuvre, notamment dans le contexte du système hors sol. Ce dernier, rencontré généralement chez les petits et moyens troupeaux, implique des variations trop importantes au niveau de l'ensemble des paramètres zootechniques associés à l'élévation cellulaire.

Tableau 7. Relation entre CCI moyens (x1000 cell. /mL) et caractéristiques des machines à traire adoptées

\begin{tabular}{|c|c|c|c|}
\hline $\begin{array}{c}\text { Paramètres } \\
\text { (par rapport à la norme) }\end{array}$ & Effectif & $\%$ & CCI moyens \\
\hline \multicolumn{4}{|l|}{ Niveau de vide } \\
\hline Inférieur & 52 & 19 & $900 \pm 1208$ \\
\hline Conforme & 53 & 19 & $933 \pm 1275$ \\
\hline Supérieur & 169 & 62 & $904 \pm 1227$ \\
\hline Total $(*)$ & 274 & 100 & \\
\hline \multicolumn{4}{|l|}{ Fréquence de pulsation } \\
\hline Inférieure & 43 & 16 & $697 \pm 1129$ \\
\hline Conforme & 39 & 14 & $1292 \pm 1705$ \\
\hline Supérieure & 192 & 70 & $879 \pm 1160$ \\
\hline Total $(*)$ & 274 & 100 & \\
\hline \multicolumn{4}{|l|}{ Rapport de pulsation } \\
\hline Inférieur & 5 & 3 & $877 \pm 1306$ \\
\hline Conforme & 118 & 79 & $908 \pm 1119$ \\
\hline Supérieur & 27 & 18 & $829 \pm 1067$ \\
\hline Total $(*)$ & 150 & 100 & \\
\hline \multicolumn{4}{|l|}{ Décalage } \\
\hline Conforme & 122 & 81 & $794 \pm 1020$ \\
\hline Supérieur & 28 & 19 & $1324 \pm 1532$ \\
\hline Total $(*)$ & 150 & 100 & \\
\hline
\end{tabular}




\begin{tabular}{cccc}
\hline État Manchons-trayeurs & & & \\
Mauvais & 86 & 29 & $1163 \pm 1589$ \\
Moyen & 162 & 55 & $882 \pm 1210$ \\
Bon & 49 & 16 & $666 \pm 910$ \\
Total $(*)$ & 297 & 100 & \\
\cline { 1 - 3 } État Tuyauterie & \multicolumn{3}{c}{} \\
Mauvais & 29 & 10 & $598 \pm 596$ \\
Moyen & 183 & 62 & $1019 \pm 1453$ \\
Bon & 85 & 28 & $844 \pm 1108$ \\
\hline Total $(*)$ & 297 & 100 & \\
\hline
\end{tabular}

(*) Les effectifs donnés diffèrent d'un paramètre à l'autre, compte tenu du manque de certaines données (non relevées).

\section{b. Pratiques de traite et leur effet sur les CCI}

L'influence de la traite sur l'incidence des mammites a été étudiée par divers auteurs. D'après Roussel \& Ribaud [42], dans leur étude, l'absence de nettoyage et de désinfection après la traite d'une vache est associée à une augmentation du risque de mammites bovines.

Tableau 8. Relation entre CCI moyens (x1000 cell. $/ \mathrm{mL})$ et pratiques de traite

\begin{tabular}{|c|c|c|c|}
\hline Paramètre & Effectif & $\%$ & CCI moyens \\
\hline \multicolumn{4}{|l|}{ Lavage Mamelle } \\
\hline Eau seulement & 129 & 43 & $1007 \pm 1391$ \\
\hline Eau et lavette collective & 117 & 39 & $934 \pm 1319$ \\
\hline Eau et savon & 29 & 10 & $1034 \pm 1125$ \\
\hline $\mathrm{Au} \mathrm{Sec}$ & 22 & 8 & $288 \pm 495$ \\
\hline Total & 297 & 100 & \\
\hline \multicolumn{4}{|l|}{ Zone mammaire nettoyée } \\
\hline Trayons & 116 & 39 & $846 \pm 1162$ \\
\hline Corps de mamelle & 147 & 50 & $946 \pm 1355$ \\
\hline Totalité de mamelle & 34 & 11 & $1127 \pm 1271$ \\
\hline Total & 297 & 100 & \\
\hline \multicolumn{4}{|l|}{ Essuyage Trayons } \\
\hline Non & 277 & 93 & $904 \pm 1240$ \\
\hline Oui & 20 & 7 & $1258 \pm 1690$ \\
\hline Total & 297 & 100 & \\
\hline \multicolumn{4}{|l|}{ Élimination Premiers jets } \\
\hline Non & 201 & 68 & $872 \pm 1189$ \\
\hline Au sol & 76 & 25 & $1256 \pm 1742$ \\
\hline Récipient & 20 & 7 & $242 \pm 297$ \\
\hline Total & 297 & 100 & \\
\hline \multicolumn{4}{|l|}{ Pose Gobelets-trayeurs } \\
\hline Indirecte & 51 & 17 & $898 \pm 1303$ \\
\hline Directe & 246 & 83 & $934 \pm 1264$ \\
\hline Total & 297 & 100 & \\
\hline \multicolumn{4}{|l|}{ Égouttage } \\
\hline Mécanique & 259 & 87 & $917 \pm 1274$ \\
\hline Manuel & 26 & 9 & $841 \pm 1054$ \\
\hline Les deux & 12 & 4 & $1354 \pm 1660$ \\
\hline
\end{tabular}




\begin{tabular}{|c|c|c|c|}
\hline Total & 297 & 100 & \\
\hline \multicolumn{4}{|l|}{ Surtraite } \\
\hline Oui & 133 & 45 & $907 \pm 1205$ \\
\hline Non & 164 & 55 & $944 \pm 1323$ \\
\hline Total & 297 & 100 & \\
\hline \multicolumn{4}{|c|}{ Dépose Gobelets-trayeurs } \\
\hline Arrachage & 161 & 54 & $1054 \pm 1496$ \\
\hline Gravité & 46 & 16 & $921 \pm 1030$ \\
\hline Les deux & 90 & 30 & $706 \pm 990$ \\
\hline Total & 297 & 100 & \\
\hline \multicolumn{4}{|c|}{ Désinfection Trayons } \\
\hline Non & 226 & 76 & $1045 \pm 1432$ \\
\hline Oui & 71 & 24 & $554 \pm 754$ \\
\hline Total & 297 & 100 & \\
\hline
\end{tabular}

Le « Mauvais » État des «Manchons-Trayeurs », le nettoyage avec de « l'Eau seulement» ou avec du «Savon» de la «Totalité de la mamelle », «l'Élimination des premiers jets » «Au sol», la «Dépose des Gobelets-Trayeurs » par «Arrachage », l'égouttage double (mécanique et manuel) et la «Non» «Désinfection des trayons », ont révélé les moyennes des CCI les plus élevées (Tableau 8).

Toutefois, les résultats de l'analyse de la variance des CCI en fonction des pratiques de traite considérées ont dévoilé que la quasi-totalité des paramètres étudiés sont non significatifs. Une constatation mérite d'être signalée concernant le phénomène de surtraite. Ce dernier a révélé des CCI proches, quelque soit le cas (avec ou sans surtraite). Un tel résultat est à considérer avec prudence, étant donné que le phénomène en question occasionne des lésions des trayons et contribue, par conséquent, à l'augmentation des fréquences de mammites [43].

\section{CONCLUSION}

L'étude des relations entre les comptages cellulaires, d'une part, les caractéristiques morphologiques et hygiéniques des vaches laitières ainsi que diverses conditions d'élevage et de traite, d'autre part, a mis en évidence certains facteurs de risque liés aux mammites bovines dans le contexte hors sol étudié en milieu semi-aride de la Tunisie littorale.

La conformation des mamelles (notamment Profondeur Pis), la propreté des vaches et de l'aire de couchage sont les facteurs essentiels ayant montré des effets significatifs sur la variation des comptages cellulaires individuels. Il est probable que d'autres facteurs, non considérés dans cette étude, soient en cause, tels que l'hygiène du trayeur, le respect d'ordre de traite des vaches infectées, la gestion du lait mammiteux, la réforme des vaches, ... L'étude d'autres facteurs de risque liés aux conditions d'élevage (litière, ...) et de traite 
(fonctionnement et état des machines à traire, pratiques techniques et hygiéniques de la traite) reste donc prioritaire pour maitriser l'apparition de nouvelles infections. De même, pour les facteurs déjà étudiés, il convient de poursuivre les investigations, afin de confirmer ou d'infirmer une autre fois les résultats discernés lors de cette étude.

Cette étude a pu fournir des informations relatives à la situation sanitaire mammaire des élevages bovins laitiers hors sol dans la région d'étude tout en recherchant les orientations applicables lors des études ultérieures à propos des diagnostics épidémiologiques descriptif et analytique des infections mammaires au niveau de chaque troupeau.

En définitive, la mise en place d'un plan de gestion des mammites bovines à l'échelle nationale, ayant pour objectif de réduire les comptages cellulaires somatiques du lait, est indispensable. Ce plan nécessiterait le recours surtout aux étapes suivantes : Visite d'élevage, analyse des données de santé mammaire, diagnostics des conditions de traite et de logement, analyse de la situation, recommandations puis visites régulières.

\section{RÉFÉRENCES}

[1] Aouadi A., Contribution à l'étude des paramètres zootechniques dans les grands élevages bovins du gouvernorat de Béja. Thèse École Nationale de Médecine Vétérinaire Sidi Thabet, Tunisie, 1991, pp. 100.

[2] Barnouin J., Geromegnace N., Chassagne M., Dorr N., Sabatier P., Revue INRA Productions Animales, 1999, 12(1), 39-48.

[3] Hanzen Ch., Propédeutique de la glande mammaire : Approche Individuelle. Sémiologie et diagnostic individuel et de troupeau, Université de Liège, Belgique, 2009, R 21, pp. 30.

[4] Sérieys F., Le point sur les mammites des vaches laitières, ITEB, Paris, 1995, pp. 65.

[5] Fourichon C., Seegers H., Beaudeau F., Bareille N., 1997, Rencontres Recherches Ruminants, 4, pp. 278

[6] Kossaibati M.A., Esslemont R.J., Veterinary Journal, 1997, 154, pp. 41-51.

[7] Seegers H., Menard J. L., Fourichon C., , Rencontres Recherches Ruminants, 1997, 4, pp. 233-242.

[8] Seegers H., Fourichon C., Beaudeau F., Veterinary Resarch, 2003, 34(5), pp. 475-491.

[9] Shim E., Shanks R., Morin D., Journal of Dairy Science, 2004, 87, pp. 2702-2708.

[10] Petrovski K., Trajcev M., Buneski G., Journal of the South Africain Veterinary Association, 2006, pp. 77: 52-60.

[11] Beaudeau F., Fourichon C., Seegers H. et Bareille N., Rencontres Recherches Ruminants, 2000, 7, pp. 87-90. 
[12] Noireterre Ph., Suivi de comptages cellulaires et d'examens bactériologiques lors de mammites cliniques chez la vache laitière, Thèse de doctorat, École Nationale Vétérinaire Lyon France, 2006, pp. 98.

[13] Schaeren W., Eviter les mammites chez la vache laitière : Fiche technique destinée à la pratique, ALP actuel, n²1, Agroscope, 2006, pp. 4.

[14] Wattiaux M., Mammites: prévention et détection. Guide technique. Institut Babcock pour la Recherche et le Développement International du Secteur Laitier, 2005, pp. 5.

[15] Dohoo I.R., Meek A.H., Revue Vétérinaire Canadienne, 1982, 23(4), pp. 119-125.

[16] Dohoo I.R., Leslie K.E., Preventive Veterinary Medicine, 1991, 10(3), pp. 225-237.

[17] Harmon R.J., Journal Dairy Science, 1994, 77(7), pp. 2103-2110.

[18] Reddy L.V., Choudhuri P.C., Hamza P.A., Indian Veterinary Journal, 1998, 75, pp. 1004-1005.

[19] Rupp R., Boichard D., Bertrand C., Bazin S., Revue INRA Productions Animales, 2000, 13(4), pp. 257-267.

[20] Hanzen Ch. and Pluvinage P., Propédeutique de la glande mammaire: Approche d'élevage 1. Université de Liège, Belgique, 2008, R22, pp. 12.

[21] Barnouin J., Fayet J.C., Jay M., Brochart M., Revue Vétérinaire Canadienne, 1986 a, 27, pp. 135-145.

[22] Barnouin J., Fayet J.C., Jay M., Brochart M. et Faye B., Revue Vétérinaire Canadienne, 1986 b, 27, pp. 173-184.

[23] Bareille N., Djabri B., Beaudeau F., Seegers H., Rencontres Recherches Ruminants., 2003, 10, pp. 285-288.

[24] Delfosse C., Froidmont E., Curnel Y., Humblet M.F., Hanzen C., Bertozzi C., Bartiaux-Thill N., 2006, Rencontres Recherches Ruminants, Paris, 13, pp. 440.

[25] Simon D. \& Jean Philippe R., Guide vétérinaire d'investigation sur la santé de la glande mammaire. Réseau canadien de recherche sur la mammite bovine (RCRMB), Faculté de médecine vétérinaire, Université de Montréal, Canada, 2005, J2S 7C6, pp. 26.

[26] Slettbakk T., Jorstad A., Farver T.B., Holmes J.C., Preventive Veterinary Medicine, 1995, 24(4), pp. 235-244.

[27] Bakken G., Acta Agriculturae Scandinavica, 1981, 31(4), pp. 438-444.

[28] Coulon J.B., Dauver F., Garel JP., INRA Production Animale, 1996, 9(2), pp. 133-139.

[29] Seykora A.J. and Mc Daniel B.T., Journal of Dairy Science, 1985, 68, pp. 2087-2093.

[30] Faye B., Barnouin J., Objectivation de la propreté des vaches laitières et des stabulations : L'indice de propreté. Bulletin Technique. Centre de Recherches Zootechniques et Vétérinaires de Theix INRA, 1985, 59, pp. 61-67. 
[31] Roussel Ph., Seegers H., Serieys F., Maîtrise de la santé des troupeaux bovins, Guide d'intervention pour la maîtrise des mammites dans les troupeaux laitiers, UMT, 2011, pp. 134.

[32] Hutton CT, Fox LK, Hancock DD., Preventive Veterinary Medicine, 1991, 11(1), pp. 25-35.

[33] Lacombe JF., Edition France Agricole, 1998, pp. 189-231.

[34] Faroult B., Journées Nationales des Groupements Techniques Vétérinaires, 1990, 3, B, 353, pp.25-39.

[35] Lecler D., Gaule J.F., Le contrôle annuel pour assurer le bon fonctionnement et l'entretien de votre machine. L'Agriculteur Normand, 2008, pp. 2.

[36] Mtaallah B., Oubey Z., Hammami H., Revue Médecine Vétérinaire, 2002, 153(4), pp. 251-260.

[37] Brouillet P., Federici C., Durel L., Journées Nationales des Groupements Techniques Vétérinaires, 2002, pp. 333-338.

[38] Hillerton J., Pankey J.W., Pankey P., Journal of Dairy Research, 2002, 69, pp. 81-84.

[39] Enault C., La Machine à traire : Recherches et innovations depuis les années 1980 en vue d'améliorer la qualité du lait et la santé de la mamelle chez les vaches laitières. Faculté de Médecine de Créteil, École Nationale Vétérinaire d'Alfort France, 2008, pp. 227.

[40] Federici-Mathieu C., Godin M., Journées Nationales des Groupements Techniques Vétérinaires, Tours, 2002, pp. 369-395.

[41] Jadoul T., Qualité du lait, problèmes rencontrés par les producteurs wallons : causes et solutions. Journée d'étude : traire un lait de qualité : Une attention de tous les jours, organisée par l'Association Wallonne de l'Elevage, le Ministère de la Région Wallonne et la Direction Générale de l'Agriculture à Henri-Chapelle (Belgique), 2005, pp. 9.

[42] Roussel Ph., Ribaud D., Étude des mammites cliniques et subcliniques chez les primipares au vêlage, 2000, CR n 2003112 .

[43] Billon P., Sauvée O., Menard J.L., Gaudin V., Rencontres Recherches Ruminants., Paris, 1998, 5, pp. 305-312.

\section{How to cite this article:}

Haj Mbarek R and M'Sadak Y. Impact of zootechnical parameters on cell quality of cattle milk (semi-arid costal Tunisia). J. Fundam. Appl. Sci., 2015, 7(3), 350-363. 